\title{
Genetic diversity and epidemiology of Genogroup II noroviruses in children with acute sporadic gastroenteritis in Shanghai, China, 2012-2017
}

Lijuan Lu, Huaqing Zhong, Menghua Xu, Liyun Su, Lingfeng Cao, Ran Jia and Jin Xu* (D

\begin{abstract}
Background: Noroviruses (NoVs) are considered an important cause of acute gastroenteritis (AGE) across all age groups, especially in children under 5 years of age. We investigated the epidemiology of noroviruses in outpatient children from the Children's Hospital of Fudan University in Shanghai, China.

Methods: Stool specimens were collected between January 2012 and December 2017 from 1433 children under 5 years of age with acute gastroenteritis. All samples were analysed by conventional reverse transcription-polymerase chain reaction (RT-PCR) for genogroup II NoVs amplifying both the RNA-dependent RNA polymerase (RdRp) and partial capsid genes. The Norovirus Genotyping Tool v.2.0 (https://www.rivm.nl/mpf/typingtool/norovirus/) was used for genotyping the strains, and phylogenetic analyses were conducted by MEGA 6.0.

Results: From 2012 to 2017, GII NoVs were detected in 15.4\% (220/1433) of the samples, with the highest detection rate in children aged $7-12$ months $(19.2 \%, 143 / 746)$. The seasons with the highest prevalence of Gll NoVs infection were autumn and winter. Based on genetic analysis of RdRp, GII.Pe $(74.5 \% \%, 137 / 184)$ was the most predominant RdRp genotype from 2013 to 2017, while Gll.P4 played a dominant role in 2012 (55.6\%, 21/36). Among the capsid genotypes, the most prevalent NoV genotype from 2012 to 2017 was Gll.4 (74.1\%, 163/220). On the basis of genetic analysis of RdRp and capsid sequences, the strains were clustered into - 19 RdRp/capsid genotypes, and 12 of them were discordant, such as GII.Pe/Gll.4-Sydney_2012, GII.P12/GII.3, GIl.P7/GIl.6, GII.Pe/GII.3, and GII.P16/GIl.2. Starting with 2013, GII.Pe/GIl.4-Sydney_2012 had completely replaced the pandemic GII.P4-2006b/GII.4-2006b subtype and was detected in children across all age groups.

Conclusions: The present study shows high detection rates and the genetic diversity of circulating NoV GIl genotypes in paediatric AGE samples from Shanghai. The findings emphasize the importance of continuous molecular surveillance of emerging NoV strains.
\end{abstract}

Keywords: Norovirus, RdRp/capsid genotypes, Epidemiology, Children, Acute gastroenteritis

\footnotetext{
* Correspondence: jinxu_125@163.com

Department of Clinical Laboratory, Children's Hospital of Fudan University, 399 Wanyuan Road, Shanghai 201102, China
}

(c) The Author(s). 2019 Open Access This article is distributed under the terms of the Creative Commons Attribution 4.0 International License (http://creativecommons.org/licenses/by/4.0/), which permits unrestricted use, distribution, and reproduction in any medium, provided you give appropriate credit to the original author(s) and the source, provide a link to the Creative Commons license, and indicate if changes were made. The Creative Commons Public Domain Dedication waiver (http://creativecommons.org/publicdomain/zero/1.0/) applies to the data made available in this article, unless otherwise stated. 


\section{Background}

Despite a substantial decrease in the prevalence of gastroenteritis in recent decades, gastroenteritis remains the second most common cause of morbidity and the fourth most common cause of mortality worldwide in children under the age of 5 [1]. After rotaviruses, noroviruses are considered the second most common viral cause of acute gastroenteritis in children [2-5].

Noroviruses (NoVs) have a non-segmented positivestrand RNA genome of approximately $7.6 \mathrm{~kb}$ that contains three ORFs [6]. ORF1 encodes a polyprotein that is cleaved by the virus-encoded protease into six nonstructural proteins, including the norovirus protease and RNA-dependent RNA polymerase (RdRp). ORF2 encodes the major structural protein (VP1), and ORF3 encodes a minor structural protein (VP2) [7]. NoVs are highly diverse and divided into seven genogroups (GIGVII), of which GI, GII and GIV have been found in humans, and GII is the most prevalent genogroup in children with acute diarrhoea. GII NoVs can be subdivided into 29 genotypes or 23 genotypes based on the genetic diversity of ORF1 or ORF2, respectively [8, 9]. An increasing number of ORF1/ORF2 combination genotypes of NoVs have been appearing in many areas [10-17]. Taking into account the phylogenetic relationships of both partial ORF1 and ORF2 sequences, a dual nomenclature system for NoVs has been proposed. This dual typing approach can correctly identify genetically different NoV genotypes. In addition, combinant genotypes are better for understanding the molecular epidemiology of NoVs.

Previous studies in Shanghai mainly adopted either ORF1 or ORF2 nomenclature to understand the epidemiology of NoVs in children under 5 years of age [18-22]. Here, we adopted a dual nomenclature system based on both ORF1 and ORF2 to investigate the diversity of NoV GII genotypes in children suffering from acute gastroenteritis who visited the Children's Hospital of Fudan University in Shanghai from January 2012 to December 2017. Furthermore, we assessed the overall frequency of GII NoVs, seasonal distribution of GII NoVs, and NoV GII genotype distribution by age group.

\section{Methods}

\section{Study design}

Faecal specimens were collected from 1433 children up to 5 years old who visited the outpatient department of the Children's Hospital of Fudan University and were diagnosed with acute gastroenteritis between January 2012 and December 2017 in Shanghai. Acute gastroenteritis is defined as $\geq 3$ instances of loose stool or looser-than-normal stool within a 24-h period combined with significant changes in the faecal exterior, including a watery or thin paste texture and the presence of mucous; this definition excluded the presence of pus or blood regardless of the presence of fever [20]. Demographic information and clinical diagnoses were collected from the patients' medical histories. The study was approved by the Institutional Review Board of the Children's Hospital of Fudan University.

Stool suspensions were prepared as $10 \%(\mathrm{w} / \mathrm{v})$ in saline solution. Nucleic acid was extracted from $200 \mu \mathrm{L}$ clarified stool suspensions using a TIANamp Virus DNA/ RNA Kit (TIANGEN Biotech (Beijing) Co., Ltd.) according to the manufacturer's recommendations. The extracted genetic material was submitted to reverse transcription (RT) with a random primer using PrimeScript $^{\mathrm{TM}}$ II Reverse Transcriptase (Takara, Biotechnology (Dalian) Co., Ltd.).

cDNA was amplified by PCR for GII NoV genotyping. PCR and sequencing of NoVs were performed using primers targeting the RdRp region of ORF1 (313 bp) and partial capsid region of ORF2 (344 bp) (Table 1). PCR was performed under the following conditions: initial denaturation at $94{ }^{\circ} \mathrm{C}$ for $2 \mathrm{~min}$, followed by 35 cycles of $94{ }^{\circ} \mathrm{C}$ for $30 \mathrm{~s}, 55^{\circ} \mathrm{C}$ for $30 \mathrm{~s}, 72^{\circ} \mathrm{C}$ for $30 \mathrm{~s}$, and $72{ }^{\circ} \mathrm{C}$ for $7 \mathrm{~min}$ for a final extension. All amplified cDNA products were electrophoresed on $2.0 \%$ agarose gels containing $4 \mathrm{~S}$ GelRed and visualized by the gel doc EZ imaging system (bio-rad laboratories (Shanghai) co., ltd.). The amplification products of NoV-positive samples were subjected to nucleotide sequencing by Sangon biotech (Shanghai) co., ltd.

Sequences generated from the PCR products of each strain were analysed by using the Norovirus Genotyping Tool v.2.0 (https://www.rivm.nl/mpf/typingtool/norovirus/), where each sequence was assigned a NoV genotype. Phylogenetic analysis of the sequences in our study and of sequences from GenBank was also performed using the maximum likelihood method (Kimura twoparameter model, 1000 bootstrap replications for branch support) in MEGA 6.0.

\section{Statistical analysis}

The difference between NoV detection rates in boys and girls was compared using a two-sided chi-square test in SPSS Statistics v.19.0 (IBM Corp., Armonk, NY, USA), and a $P$-value less than 0.05 was considered statistically significant.

\section{Results}

Detection and epidemiology of GII NoVs in children

A total of 1433 stool samples were collected from 2012 to 2017 from outpatient children with acute gastroenteritis, of which 897 (62.6\%) were boys and 536 (37.4\%) 
Table 1 Primers used for Noroviruses genotyping in this study

\begin{tabular}{lllll}
\hline Primers & Nucleotide position & Polarity & Target & Sequence (5'- 3') \\
\hline G2SKF & 5058 & F & Capsid & CNTGGGAGGGCGATCGCAA \\
G2SKR & 5401 & R & Capsid & CCRCCNGCATRHCCRTTRTACAT \\
$289 \mathrm{H}$ & 4865 & R & RdRp & TGACGATTCATCATCACCATA \\
$289 \mathrm{l}$ & 4865 & $\mathrm{R}$ & RdRp & TGACGATTTCATCATCCCCGTA \\
$290 \mathrm{H}$ & 4590 & $\mathrm{~F}$ & RdRp & GATTACTCCAGGTGGGACTCCAC \\
$290 \mathrm{I}$ & 4590 & $\mathrm{~F}$ & RdRp & GATTACTCCAGGTGGGACTCAAC \\
$290 \mathrm{~J}$ & 4590 & $\mathrm{~F}$ & RdRp & GATTACTCCAGGTGGGATCAAC \\
$290 \mathrm{~K}$ & 4590 & F & RdRp & GATTACTCCAGGTGGGATCCAC \\
\hline
\end{tabular}

were girls. Among these children, 15.4\% (220/1433) were infected with the NoV GII genogroup, and the annual detection rates were $25.0 \%(36 / 144), 15.3 \%(22 / 144)$, $11.8 \%(17 / 144), 15.5 \%(41 / 265), 18.8 \%(59 / 313)$ and $10.6 \%$ (45/423) from 2012 to 2017, respectively. No significant difference $(P>0.05)$ in the NoV detection rate was observed between boys $(16.4 \%, 147 / 897)$ and girls (13.6\%, 73/536).

The overall prevalence of GII NoV infection in the different age groups ranged from 5.8 to $19.2 \%$. Children in the age group 7-12 months had the highest prevalence $(19.2 \%, 143 / 746)$, followed by children aged 13 to 24 months $(15.3 \%, 26 / 170)$ (Fig. 1). Most of the children infected with NoVs $(93.6 \%, 206 / 220)$ were aged less than 2 years. GII NoV infection was prevalent in all seasons from 2012 to 2017, and the seasons with the highest prevalence of NoV infection were autumn and winter, except for a small peak in the summer of 2012 (Fig. 2).

\section{NoV Gll genotype distribution based on the RdRp region} When the polymerase region was analysed, the large genetic diversity of the circulating NoV GII strains was observed in the $220 \mathrm{NoV}$-positive samples. GII.Pe (74.5\%, 137/184) was the predominant RdRp genotype from 2013 to 2017, while GII.P4 played a dominant role in $2012(55.6 \%, 21 / 36)$. The second most prevalent genotype varied from year to year. GII.Pe and GII.P7 were the second most prevalent genotypes in 2012 and 2014, while GII.P12 was the second most prevalent genotype in the rest of the years. GII.P17 was sequenced successfully from eight samples from 2015 to 2017. In addition, many other RdRp genotypes were detected, such as GII.Pg $(1.4 \%, 3 / 220)$, GII.P16 $(0.5 \%, 1 / 220)$ and GII.P8 $(0.5 \%, 1 / 220)$. Three GII.P4 subtypes were detected in only 2012, including GII.P4-2006b (50.0\%, 18/36), GII.P4-2006a (2.8\%, 1/ 36) and GII.P4-New_Orleans_2009 (5.6\%, 2/36) (Figs. 3 and 4a, Table 2).

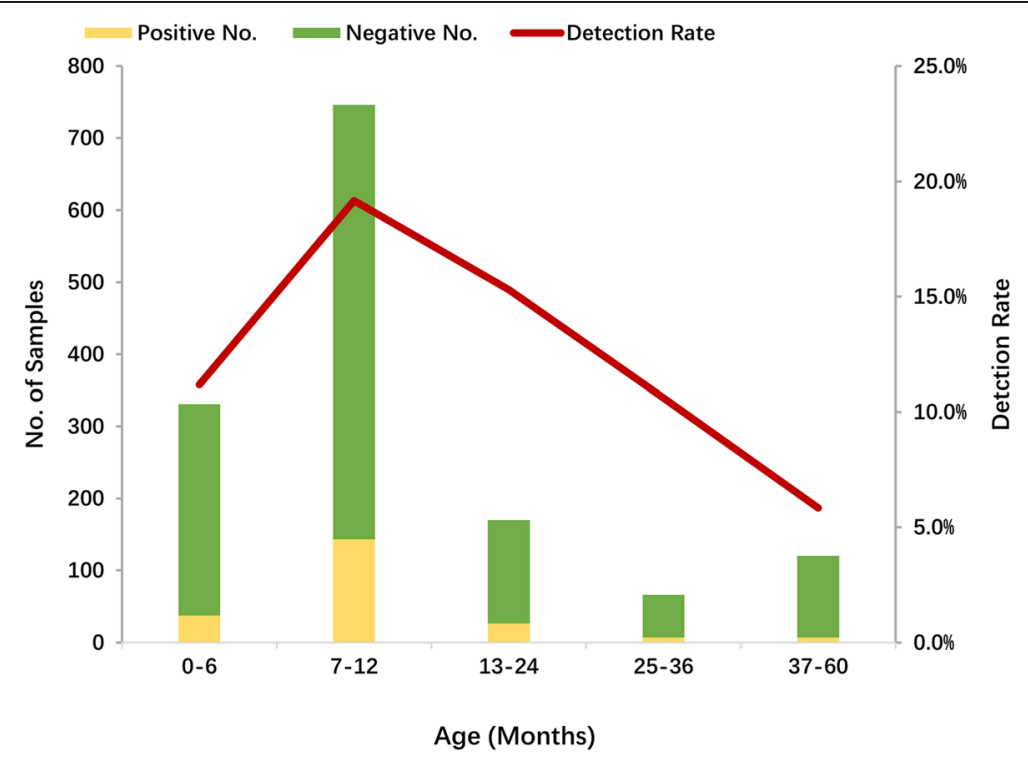

Fig. 1 Age distribution of norovirus infection in children under 5 years of age with acute gastroenteritis in Shanghai, 2012-2017 


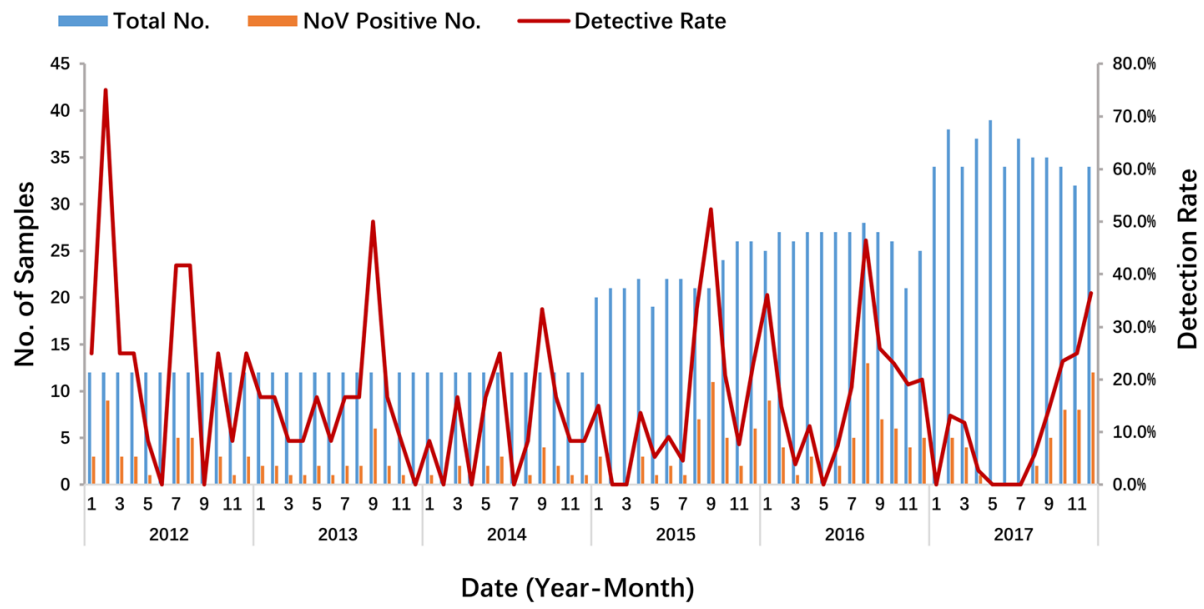

Fig. 2 Seasonal distribution of norovirus infection in children under 5 years old with acute gastroenteritis in Shanghai, 2012-2017

\section{NoV GIl genotype analysis based on the partial capsid} region

On the basis of the partial capsid region, the majority of the NoV GII strains were classified as GII.4 (73.6\%, 162/ 220) from 2012 to 2017, followed by GII.3 (15.9\%, 35/ 220). The other non-GII.4 genotypes included GII.17 (3.6\%, 8/220), GII.6 (2.7\%, 6/220), GII.1 (1.8\%, 4/220), GII.2 (0.9\%, 2/220), GII.7 (0.5\%, 1/220) and GII.8 (0.5\%, $1 / 220$ ). GII.4 was divided into three subtypes by the Norovirus Genotyping Tool v.2.0. GII.4-Sydeny_2012 $(73.9 \%, 136 / 184)$ was the most frequent GII.4 variant from 2013 to 2017, while GII.4-2006b (50.0\%, 18/36) was the main variant in 2012. Two sequences detected in samples from 2012 belonged to GII.4-New_Orleans 2009 (Figs. 3 and 4b-g, Table 2).

\section{Combination genotype of GII NoVs with both RdRp/ capsid fragment genes}

Overall, 19 kinds of $\mathrm{RdRp} /$ capsid genotypes were presented according to the dual nomenclature system of NoVs, and discordant RdRp and capsid genotypes were identified in 12 of them, including GII.Pe/GII.4Sydney_2012, GII.P12/GII.3, GII.P7/GII.6 and GII.P16/GII.2. GII.Pe/GII.4-Sydney_2012 (73.4\%, 135/ 184) was the dominant RdRp/capsid genotype in each year from 2013 to 2017, while GII.P4-2006b/GII.42006b $(44.4 \%, 16 / 36)$ was the most frequent genotype in 2012. GII.P12/GII.3 was the second most frequent combination genotype in $2013(27.3 \%, 6 / 22), 2015$ (34.1\%, 14/41), $2016(10.2 \%, 6 / 59)$ and 2017 (8.9\%, 4/ 45), while GII.Pe/GII.4-Sydney_2012 (16.7\%, 6/36) and GII.P7/GII.6 (17.6\%, 3/17) were the second most frequent genotypes in 2012 and 2014. Most of the uncommon combination genotypes were detected in 2012, for example, GII.Pe/GII.6, GII.Pg/GII.1 and GII.Pe/GII.4-2006b. In addition, GII.P17/GII.17 was only detected in eight children from 2015 to 2017 (Fig. 5).

\section{Distribution of NoV GII RdRp/capsid genotype in children of different ages}

In this study, only the GII.Pe/GII.4-Sydney_2012 genotype was distributed across all age groups in children. Almost all the NoV RdRp/capsid genotypes were detected in children aged from 7 to 12 months $(63.6 \%$, 143/220). Among these genotypes, GII.P2/GII.2, GII.Pg/ GII.1, GII.Pe/GII.6, GII.P4-2006b/GII.1, GII.P4-2006b/ GII.4-Sydney_2012, GII.P12/GII.4-Sydney_2012, GII.P7/ GII.4-Sydney_2012 and GII.Pe/GII.4-2006b were only detected in 7- to 12-month-old children. GII.P4-2006a/ GII.4-Sydney_2012， GII.P6/GII.6, GII.P7/GII.7 and GII.P8/GII.8 were only distributed in children aged from 0 to 6 months, and GII.P16/GII.2 was only detected in a child aged 60 months (Fig. 6).

\section{Discussion}

This was a long-term monitoring study of the epidemiology and molecular characteristics of NoVs in children under 5 years of age with acute sporadic gastroenteritis in Shanghai. Herein, the total detection rate of GII norovirus-positive cases (15.4\%) among outpatients was lower than that in our previous monitoring data of NoV infections in both outpatients and inpatients $[20,22]$. Although the percentage of NoV infections in 2012 (25.0\%) was similar to our previous data from 2006 to 2011, the annual detection rate of NoVs was characterized by a fluctuating reduction from 2012 to 2017 [22]. This finding may be associated with the increased publicity on how to prevent NoV infection among people because of the increasing number of outbreaks of $\mathrm{NoV}$ infection in many areas in recent years. 


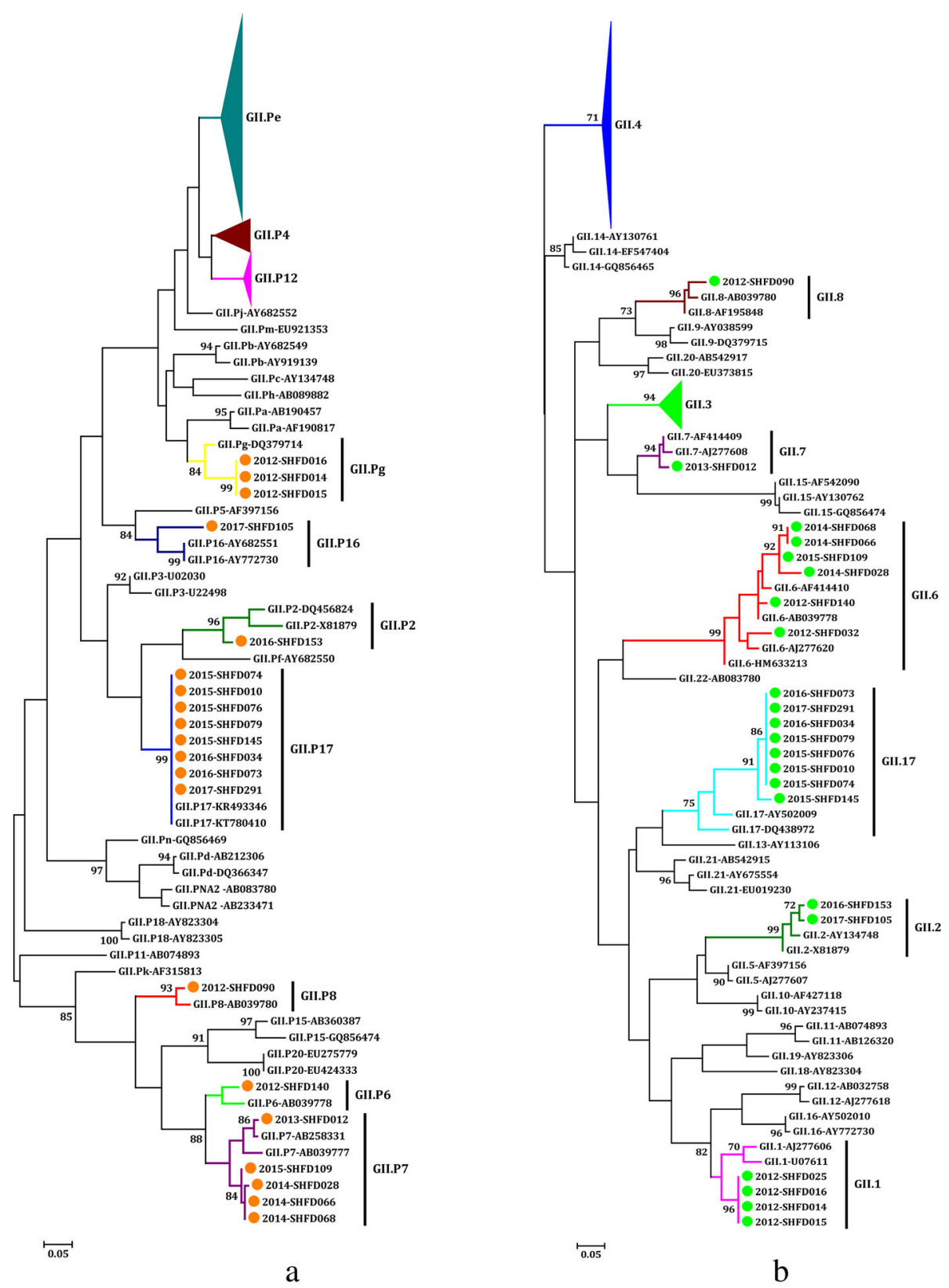

Fig. 3 Phylogenetic analysis of GIl norovirus based on the partial nucleotide sequences of the viral polymerase (a) and capsid regions (b). The NoV strains detected in this study are indicated by coloured dots. References NoV genotypes are labelled according to GenBank with their respective accession numbers. The trees were constructed in MEGA 6.0 through the maximum likelihood method using the Kimura 2-parameter model. The bootstrap values (1000 replicates) are indicated in the phylogenetic tree, and values less than 70\% are not represented

From 2012 to 2017, children with GII NoV infection were mainly aged less than 2 years, which is consistent with our previous study and other studies [20, 22-25]. No significant difference in NoV detection rates was found in girls and boys. This finding may imply that gender is not a predisposing factor for NoV infection in children less than 5 years old. In accordance with previous studies that concluded that noroviruses mainly peaked in cold seasons [20, 22, 26-28], our study demonstrated that most NoV infections were detected in autumn and winter. However, in some other areas, NoV-associated diarrhoea had a summer peak or no apparent seasonal peak, which may be connected with an increase in contaminated water and food or other unknown reasons [21, 29].

A great diversity in NoV GII genotypes was identified on the basis of the RdRp region. Among the NoV-positive cases, GII.P4 was the predominant NoV genotype in 


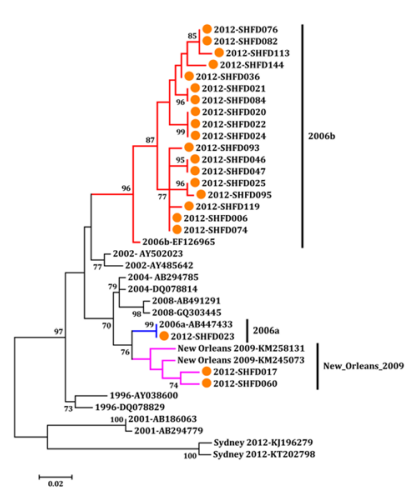

$\mathrm{a}$

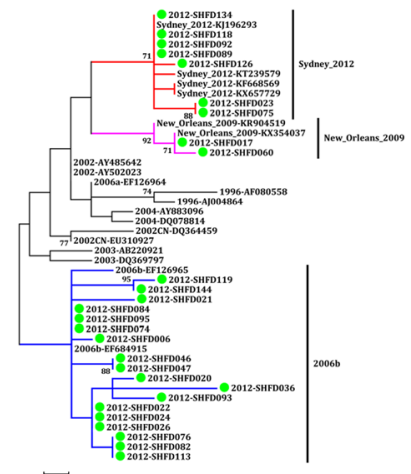

b

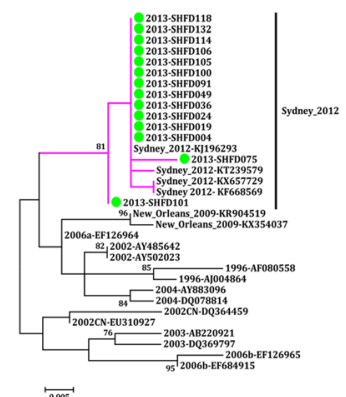

c

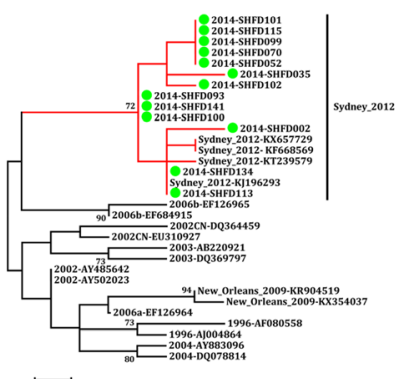

$\stackrel{.005}{0.5}$

d

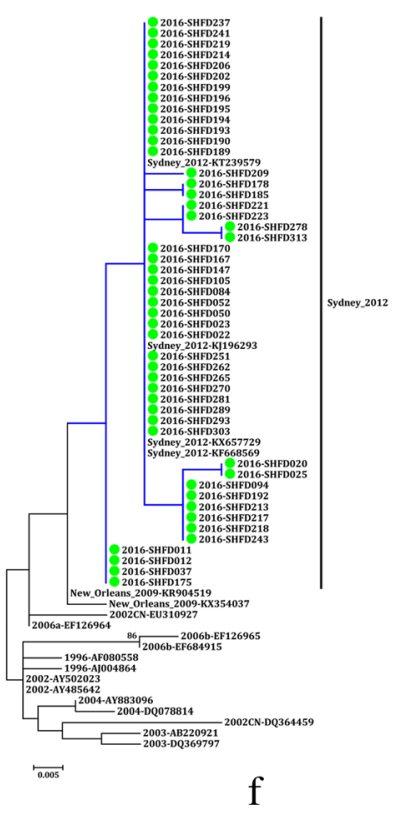

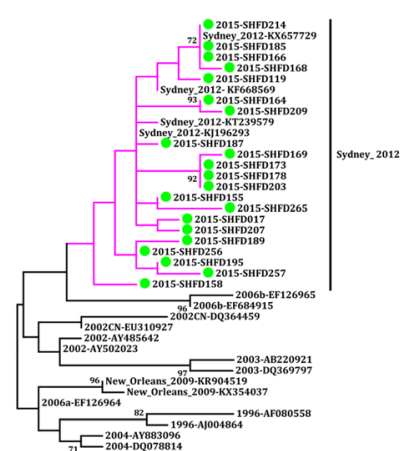

'.o.s'

e

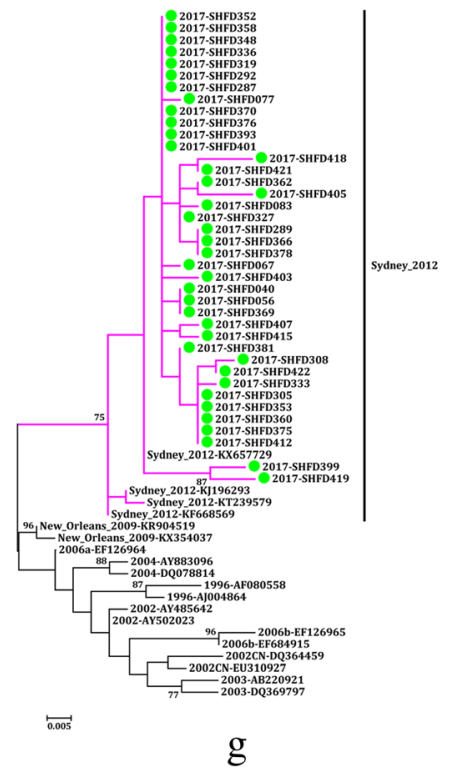

Fig. 4 Phylogenetic analysis of Gll.4 variants based on the partial nucleotide sequences of the viral polymerase (a: 2012) and capsid regions (b-g: 2012-2017). References NoV genotypes are labelled according to GenBank with their respective accession numbers. The trees were constructed in MEGA 6.0 through the maximum likelihood method using the Kimura 2-parameter model. The bootstrap values (1000 replicates) are indicated in the phylogenetic tree, and values less than $70 \%$ are not represented 
Table 2 NoV Gll genotypes distribution according to the RdRp region of ORF1 or partial region of ORF2 in Children with acute gastroenteritis under 5 years in Shanghai, 2012-2017

\begin{tabular}{|c|c|c|c|c|c|c|c|}
\hline \multirow[t]{2}{*}{ Genotypes } & 2012 & 2013 & 2014 & 2015 & 2016 & 2017 & Total \\
\hline & \multicolumn{7}{|l|}{$\mathrm{n}(\mathrm{m} \%)$} \\
\hline \multicolumn{8}{|l|}{ RdRp region of ORF1 } \\
\hline GII.P2 & - & - & - & - & $1(1.7)$ & & $1(0.5)$ \\
\hline GII.P4-2006a & $1(2.8)$ & - & - & - & - & - & $1(0.5)$ \\
\hline Gll.P4-2006b & $18(50.0)$ & - & - & - & - & - & 18(8.1) \\
\hline Gll.P4-New_Orleans_2009 & $2(5.6)$ & - & - & - & - & - & $2(0.9)$ \\
\hline Gll.P6 & $1(2.8)$ & - & - & - & - & - & $1(0.5)$ \\
\hline Gll.P7 & - & $1(4.5)$ & $3(17.6)$ & $1(2.4)$ & & & $5(2.2)$ \\
\hline Gll.P8 & $1(2.8)$ & - & - & - & - & - & $1(0.5)$ \\
\hline GII.P12 & $2(5.5)$ & $6(27.3)$ & $1(5.9)$ & $14(34.2)$ & $7(11.9)$ & $4(8.9)$ & $34(15.4)$ \\
\hline Gll.P16 & - & - & - & - & - & $1(2.2)$ & $1(0.5)$ \\
\hline Gll.P17 & - & - & - & $5(12.2)$ & 2(3.4) & $1(2.2)$ & $8(3.6)$ \\
\hline Gll.Pe & $8(22.2)$ & $15(68.2)$ & $13(76.5)$ & $21(51.2)$ & 49(83.0) & $39(86.7)$ & 145(65.9) \\
\hline Gll.Pg & $3(8.3)$ & - & - & - & - & - & $3(1.4)$ \\
\hline Total & $36(100)$ & $22(100)$ & $17(100)$ & $41(100)$ & $59(100)$ & $45(100)$ & $220(100)$ \\
\hline \multicolumn{8}{|l|}{ Partial region of ORF2 } \\
\hline Gll.1 & $4(11.1)$ & - & - & - & - & - & $4(1.8)$ \\
\hline Gll.2 & - & - & - & - & $1(1.7)$ & $1(2.2)$ & $2(0.9)$ \\
\hline Gll.3 & $2(5.6)$ & $7(31.8)$ & $1(5.9)$ & 14(34.2) & $7(11.9)$ & $4(8.9)$ & $35(15.9)$ \\
\hline Gll.4-2006b & $18(50.0)$ & - & - & - & - & - & $18(8.2)$ \\
\hline Gll.4-Sydney_2012 & 7(19.4) & 14(63.6) & $13(76.5)$ & $21(51.2)$ & 49(83.0) & 39(86.7) & 143(65.0) \\
\hline Gll.4-New_Orleans_2009 & $2(5.6)$ & - & - & - & - & - & $2(0.9)$ \\
\hline Gll.6 & 2(5.6) & - & $3(17.6)$ & $1(2.4)$ & - & - & $6(2.7)$ \\
\hline Gll.7 & - & $1(4.6)$ & - & - & - & - & $1(0.5)$ \\
\hline Gll.8 & $1(2.7)$ & - & - & - & - & - & $1(0.5)$ \\
\hline Gll.17 & - & - & - & $5(12.2)$ & 2(3.4) & $1(2.2)$ & $8(3.6)$ \\
\hline
\end{tabular}

$\mathrm{n}$ : NoV positive numbers

$\mathrm{m}$ : Constituent ratio of each genotype

2012, which is consistent with our previous data on outpatient children from 2010 to 2011 and western China from 2010 to 2013 [17, 20]. It is interesting to note that the prevalence of GII.P4 abruptly disappeared after 2012, while GII.Pe became the obviously dominant genotype circulating in children under 5 years of age with acute sporadic gastroenteritis from 2013 to 2017. This unexpected increase in GII.Pe from 2013 was also found in Huzhou, China [30]. Based on these results, we speculated that GII.Pe, first detected in the norovirus outbreak of 2008 in Victoria, Australia, has obviously replaced GII.P4 as the leading RdRp genotype in children with sporadic gastroenteritis in Shanghai since 2013 [31]. However, no GII.Pe was detected in Suzhou (China) and western China in 2013 [17, 32]. Close attention should be paid to the spread of this genotype in China in the future. It was surprising that GII.P12 became the second main $\mathrm{RdRp}$ genotype instead of GII.Pb from 2012 to
2017. No GII.Pb was detected in this study, although it was the second most predominant RdRp genotype from 2010 to 2011 in Shanghai [20].

Since 2014, GII.17 has become increasingly popular in several major cities in mainland China and other areas in Asia [33-36]. However, only eight GII.P17/GII.17 strains were detected from 2015 to 2017 in our study. The same detection rate of GII.P17/GII.17 in children was also reported in Huzhou and Shanghai [30, 36]. However, adults were more susceptible to GII.P17/ GII.17 than children [30, 36]. The reason for this different susceptibility to infection between diverse age groups is still unclear, and further studies need to be conducted to explore the mechanism of this difference.

Similar to the NoV RdRp genotypes, diverse NoV GII genotypes were detected on the basis of the partial capsid gene. Similar to data from Korea (2013-2015), Japan (2008-2014), Chongqing and Suzhou in China (2010- 


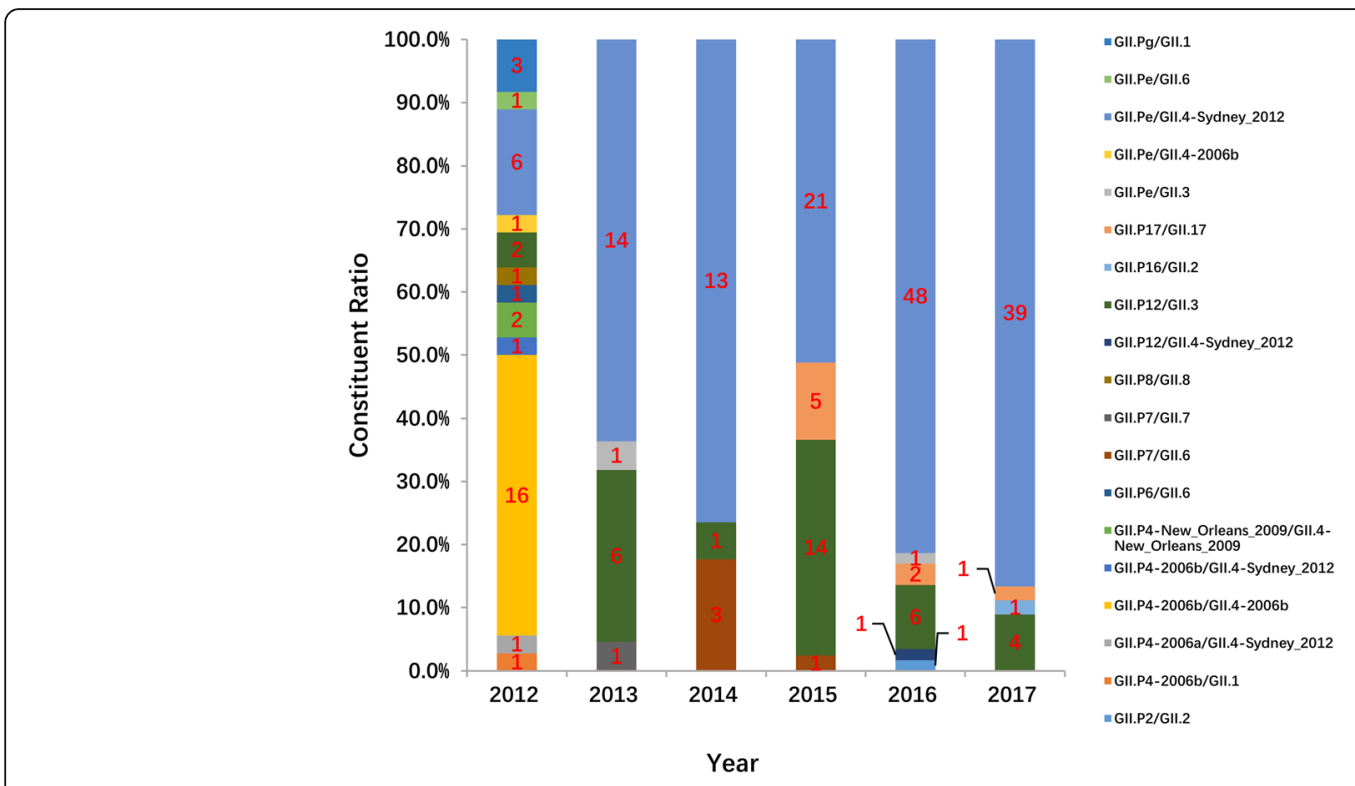

Fig. 5 Distribution of NoV RdRp/capsid combination genotypes in children under 5 years old with acute gastroenteritis in Shanghai, 2012-2017

2013), Lusaka Province in Zambia (2012-2013) and Vietnam (2012-2015), GII.4 was the predominant capsid genotype spreading among children from 2012 to 2017 $[17,32,37-40]$. The differences in the predominant genotypes determined by the two sequence-based typing methods indicated the importance of genotyping NoVs simultaneously by both capsid and RdRp genes, which could assist us in comprehensively understanding the epidemiology and evolution of NoVs. Although GII.42006b was still the predominant GII.4 variant, GII.4-Sydney_2012 became the main and only GII.4 subtype from 2013 to 2017. GII.4-Sydney_2012 was first reported in Australia, and it has been the most prevalent variant among children worldwide since 2012 [41]. As reported in Bangladesh (2010-2014), Chongqing (2010-2013) and Jiangsu (2010-2013) in China, in Japan (2008-2012) and in Vietnam (2012-2015), GII.3 was the second most prevalent capsid genotype in our study [17, 32, 38, 40, 42].

The analysis of combined NoV GII genotypes conducted in this study demonstrated that 19 different $\mathrm{RdRp} /$ capsid genotypes were determined and that 12 of them were distinct in RdRp genotypes and capsid genotypes. All of the discordant RdRp/capsid genotypes were suspected to be recombinant strains, and most of them have been reported elsewhere [14, 16, 17, 23, 37, 41]. Although more analysis of the junction of ORF1 and ORF2 is needed to confirm the recombination site in our study, the results clearly suggest that this phenomenon is very common in NoVs, as observed elsewhere. Furthermore, we observed a change in the circulation pattern of the RdRp/capsid-genotyped strains, with GII.P4-2006b/GII.4-2006b being predominant in 2012,

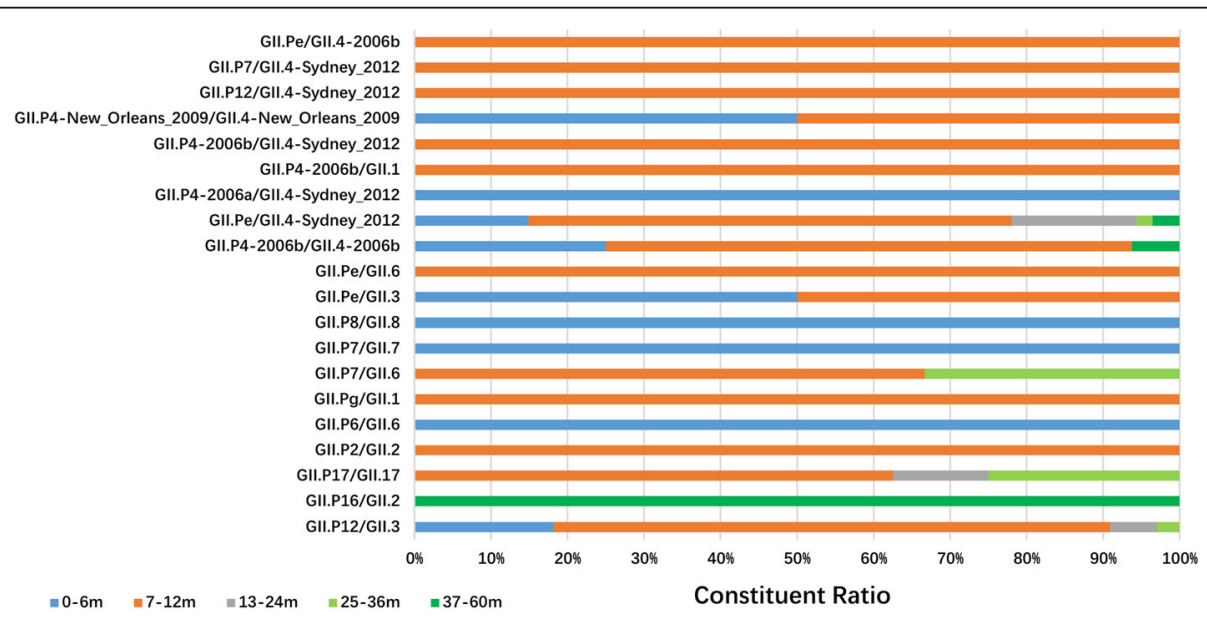

Fig. 6 Distribution of NoV RdRp/capsid combination genotypes in children of different ages with acute gastroenteritis in Shanghai, 2012-2017 
followed by an emergence and predominance of GII.Pe/GII.4-Sydney_2012 starting in 2013. GII.Pe/ GII.4-Sydney_2012, first reported in Australia in 2012, has been widespread in South Africa (2012-2013), Iran (2015-2016), Botswana (2013-2015), Korea (2013) and some cities in China (2012-2015) since then [23, 31, 37, 41, 43-46]. It was surprising that, starting with 2013, GII.Pe/GII.4-Sydney_2012 had completely replaced the pandemic GII.P4-2006b/ GII.4-2006b subtype, and close attention should be paid to the prevalence of this genotype.

The second most predominant $\mathrm{RdRp} /$ capsid genotype in 2013 and from 2015 to 2017, GII.P12/GII.3 was also reported as the main NoV genotype in Chongqing (2011-2013), China [17]. Interestingly, this genotype was mainly observed in the Asia-Pacific region, implying that the GII.P12/GII.3 pandemic may have regional characteristics [17, 47, 48]. GII.P7/GII.6, as the second most prevalent genotype in 2014, was reported in a few cases elsewhere $[46,49]$. In addition, many other rare $\mathrm{RdRp} /$ capsid strains, such as GII.Pe/GII.3 and GII.P16/GII.2, were detected in our study. Among these strains, the GII.P16/GII.2 strain was observed only in 2017; however, this strain has become the main genotype in Japan, France, Hong Kong, and Taiwan, as well as several other cities in China during 2016 and 2017 [17, 50-54]. Consequently, it is necessary to continuously monitor those rare strains in Shanghai.

In epidemiological investigations of children of different ages, the distribution of NoV RdRp/capsid genotypes varied in the different age groups. In our study, GII.Pe/ GII.4-Sydney_2012 was detected in all age groups, while other genotypes were not. Some genotypes were only detected in one age group. These results may imply that the infection of some NoV RdRp/capsid genotypes was age specific. However, more and longer surveillance of the epidemiology of NoVs in children of different ages should be conducted to illustrate this phenomenon.

\section{Conclusions}

In conclusion, our study has demonstrated the epidemiology and great genetic diversity of NoV GII combination genotypes in outpatient children less than 5 years old in Shanghai from 2012 to 2017. Many discordant $\mathrm{RdRp} /$ capsid genotypes were detected in our study and were reported for the first time in this area. Thus, continuous monitoring of $\mathrm{NoV} \mathrm{RdRp} /$ capsid genotypes is needed to predict the emergence of new pandemic strains and guide the selection of norovirus vaccine strains in Shanghai.

\section{Abbreviations}

AGE: Acute gastroenteritis; NoVs: Noroviruses; ORFs: Open reading frames: RdRp: RNA-dependent RNA polymerase

\section{Acknowledgements}

We thank the participants for their contributions to this study.

\section{Authors' contributions}

$J X$ and $L L$ conceived of and designed the study. $L J L$ acquired and analyzed all the data, interpreted the data, drafted the manuscript and obtained funding. HQZ, MHX, LYS, LFC and RJ acquired the clinical data and coordinated in the design of the study. All authors provided critical comments or revisions, approved the final version of the article.

\section{Funding}

This work was supported by Shanghai Municipal Population and Family Planning Commission (20154Y0139). The funding body played no role in the design of the study, data collection, data analysis, interpretation of data, and writing of the manuscript.

\section{Availability of data and materials}

The datasets used in the current study are available from the corresponding author on reasonable request.

\section{Ethics approval and consent to participate}

This study was approved by the Institutional Review Board of Children's Hospital of Fudan University. Because fecal specimens enrolled in this study were the remaining samples after routine examination, consent was not sought from the parent or legal guardians of the children.

\section{Consent for publication}

Not applicable.

\section{Competing interests}

The authors declare that they have no competing interests.

Received: 10 May 2019 Accepted: 5 August 2019

Published online: 22 August 2019

\section{References}

1. Ben L, Robert A, Ralph B, Mary E, Kim G, Roger G, et al. Global Burden of Norovirus and Prospects for Vaccine Development. 2015. https://www.cdc. gov/norovirus/downloads/global-burden-report.pdf. Accessed Aug 2015.

2. Ahmed $S M$, Hall AJ, Robinson AE, Verhoef $L$, Premkumar P, Parashar UD, et al. Global prevalence of norovirus in cases of gastroenteritis: a systematic review and meta-analysis. Lancet Infect Dis. 2014;14(8):725-30.

3. Patel MM, Widdowson MA, Glass RI, Akazawa K, Vinjé J, Parashar UD. Systematic literature review of role of noroviruses in sporadic gastroenteritis. Emerg Infect Dis. 2008;14(8):1224-31.

4. Anderson EJ. Prevention and treatment of viral diarrhea in pediatrics. Expert Rev Anti-Infect Ther. 2010;8(2):205-17.

5. Lopman BA, Steele D, Kirkwood CD, Parashar UD. The vast and varied global burden of norovirus: prospects for prevention and control. PLoS Med. 2016; 13(4):e1001999.

6. Jiang $X$, Wang M, Wang K, Estes MK. Sequence and genomic organization of Norwalk virus. Virology. 1993;195(1):51-61.

7. Thorne LG, Goodfellow IG. Norovirus gene expression and replication. J Gen Virol. 2014;95(Pt 2):278-91.

8. Vinjé J. Advances in laboratory methods for detection and typing of norovirus. J Clin Microbiol. 2015;53(2):373-81.

9. Hoa Tran TN, Trainor E, Nakagomi T, Cunliffe NA, Nakagomi O. Molecular epidemiology of noroviruses associated with acute sporadic gastroenteritis in children: global distribution of genogroups, genotypes and Gll.4 variants. J Clin Virol. 2013;56(3):185-93.

10. van Beek J, de Graaf M, Al-Hello H, Allen DJ, Ambert-Balay K, Botteldoorn N, et al. Molecular surveillance of norovirus, 2005-16: an epidemiological analysis of data collected from the NoroNet network. Lancet Infect Dis. 2018;18(5):545-53.

11. Eden JS, Tanaka MM, Boni MF, Rawlinson WD, White PA. Recombination within the pandemic norovirus Gll.4 lineage. J Virol. 2013;87(1 1):6270-82.

12. Kroneman A, Vega E, Vennema H, Vinjé J, White PA, Hansman G, et al. Proposal for a unified norovirus nomenclature and genotyping. Arch Virol. 2013;158(10):2059-68.

13. Di Felice E, Mauroy A, Pozzo FD, Thiry D, Ceci C, Di Martino B, et al. Bovine noroviruses: a missing component of calf diarrhoea diagnosis. Vet J. 2016; 207:53-62. 
14. Supadej K, Khamrin P, Kumthip K, Kochjan P, Yodmeeklin A, Ushijima H, et al. Wide variety of recombinant strains of norovirus Gll in pediatric patients hospitalized with acute gastroenteritis in Thailand during 2005 to 2015. Infect Genet Evol. 2017:52:44-51.

15. Andrade JSR, Fumian TM, Leite JPG, Assis MR, Bello G, Mir D, et al. Detection and molecular characterization of emergent GII.P17/GIl.17 norovirus in Brazil, 2015. Infect Genet Evol. 2017;51:28-32.

16. Fu JG, Shi C, Xu C, Lin Q, Zhang J, Yi QH, et al. Outbreaks of acute gastroenteritis associated with a re-emerging GII.P16-GIl.2 norovirus in the spring of 2017 in Jiangsu, China. PLoS One. 2017;12(12):e0186090.

17. Lu QB, Huang DD, Zhao J, Wang HY, Zhang XA, Xu HM, et al. An increasing prevalence of recombinant GII norovirus in pediatric patients with diarrhea during 2010-2013 in China. Infect Genet Evol. 2015;31:48-52.

18. Xue C, Pan L, Zhu W, Wang Y, Fu H, Cui C, et al. Molecular epidemiology of genogroup II norovirus infections in acute gastroenteritis patients during 2014-2016 in Pudong new area, Shanghai, China. Gut Pathog. 2018;10:7.

19. Pan L, Xue C, Fu H, Liu D, Zhu L, Cui C, et al. The novel norovirus genotype Gll.17 is the predominant strain in diarrheal patients in Shanghai, China. Gut Pathog. 2016:8:49.

20. Lu L, Jia R, Zhong $H$, Xu M, Su L, Cao L, et al. Molecular characterization and multiple infections of rotavirus, norovirus, sapovirus, astrovirus and adenovirus in outpatients with sporadic gastroenteritis in Shanghai, China, 2010-2011. Arch Virol. 2015;160(5):1229-38

21. Zeng M, Xu X, Zhu C, Chen J, Zhu Q, Lin S, et al. Clinical and molecular epidemiology of norovirus infection in childhood diarrhea in China. J Med Virol. 2012;84(1):145-51.

22. Lu L, Zhong H, Xu M, Su L, Cao L, Dong N, et al. Molecular epidemiology of human calicivirus infections in children with acute diarrhea in Shanghai: a retrospective comparison between inpatients and outpatients treated between 2006 and 2011. Arch Virol. 2014;159(7):1613-21.

23. Farsi M, Roodbari F, Nejati B, Arashkia A, Jalilvand S, Nateghian A, et al. Prevalence and genetic diversity of norovirus genogroup || in children less than 5 years of age with acute gastroenteritis in Tehran, Iran. Med Microbiol Immunol. 2018;207(3-4):201-10.

24. Hassine-Zaafrane M, Sdiri-Loulizi K, Kaplon J, Salem IB, Pothier P, Aouni M, et al. Prevalence and genetic diversity of norovirus infection in Tunisian children (2007-2010). J Med Virol. 2013;85(6):1100-10.

25. Esteves A, Nordgren J, Tavares C, Fortes F, Dimbu R, Saraiva N, et al. Genetic diversity of norovirus in children under 5 years of age with acute gastroenteritis from Angola. Epidemiol Infect. 2018;146(5):551-7.

26. Xue Y, Pan H, Hu J, Wu H, Li J, Xiao W, et al. Epidemiology of norovirus infections among diarrhea outpatients in a diarrhea surveillance system in Shanghai, China: a cross-sectional study. BMC Infect Dis. 2015;15:183.

27. Ahmed SM, Lopman BA, Levy K. A systematic review and meta-analysis of the global seasonality of norovirus. PLoS One. 2013;8(10):e75922.

28. Tan D, Deng L, Wang M, Li X, Ma Y, Liu W. High prevalence and genetic diversity of noroviruses among children with sporadic acute gastroenteritis in Nanning City, China, 2010-2011. J Med Virol. 2015;87(3):498-503.

29. Siafakas N, Zerva L, Hatzaki D, Lebessi E, Chronopoulou G, Paraskakis I, et al. Molecular epidemiology of noroviruses in children in South Greece, 20132015. J Med Virol. 2018;90(11):1703-11.

30. Zhang P, Chen L, Fu Y, Ji L, Wu X, Xu D, et al. Clinical and molecular analyses of norovirus-associated sporadic acute gastroenteritis: the emergence of Gll.17 over Gll.4, Huzhou, China, 2015. BMC Infect Dis. 2016; 16(1):717.

31. Bruggink LD, Dunbar NL, Marshall JA. Emergence of Gll.E as a major ORF 1 norovirus genotype and its associated ORF 2 Gll.4 variant forms. Infect Genet Evol. 2014;22:157-63.

32. Fu JG, Ai J, Zhang J, Wu QB, Qi X, Ji H, et al. Molecular epidemiology of genogroup II norovirus infection among hospitalized children with acute gastroenteritis in Suzhou (Jiangsu, China) from 2010 to 2013. J Med Virol. 2016;88(6):954-60.

33. Xue L, Dong R, Wu Q, Li Y, Cai W, Kou X, et al. Molecular epidemiology of noroviruses associated with sporadic gastroenteritis in Guangzhou, China, 2013-2015. Arch Virol. 2016;161(5):1377-84.

34. Lu J, Sun L, Fang L, Yang F, Mo Y, Lao J, et al. Gastroenteritis outbreaks caused by norovirus Gll.17, Guangdong Province, China, 2014-2015. Emerg Infect Dis. 2015;21(7):1240-2.

35. Han J, Ji L, Shen Y, Wu X, Xu D, Chen L. Emergence and predominance of norovirus Gll.17 in Huzhou, China, 2014-2015. Virol J. 2015;12:139.
36. Chen H, Qian F, Xu J, Chan M, Shen Z, Zai S, et al. A novel norovirus Gll.17 lineage contributed to adult gastroenteritis in Shanghai, China, during the winter of 2014-2015. Emerg Microb Infect. 2015;4(11):e67.

37. Dang Thanh H, Than VT, Nguyen TH, Lim I, Kim W. Emergence of norovirus Gll.17 variants among children with acute gastroenteritis in South Korea. PLoS One. 2016;11(5):e0154284.

38. Nakamura N, Kobayashi S, Minagawa H, Matsushita T, Sugiura W, Iwatani Y. Molecular epidemiology of enteric viruses in patients with acute gastroenteritis in Aichi prefecture, Japan, 2008/09-2013/14. J Med Virol. 2016:88(7):1180-6.

39. Howard LM, Mwape I, Siwingwa M, Simuyandi M, Guffey MB, Stringer JS, et al. Norovirus infections in young children in Lusaka Province, Zambia: clinical characteristics and molecular epidemiology. BMC Infect Dis. 2017;17(1):92.

40. Hoa-Tran TN, Nakagomi O, Dao AT, Nguyen AT, Agbemabiese CA, Vu HM, et al. Molecular epidemiology of noroviruses detected in Vietnamese children with acute gastroenteritis from 2012 to 2015. J Med Microbiol. 2017;66(1): 34-45.

41. Bruggink LD, Moselen JM, Marshall JA. Genotype analysis of noroviruses associated with gastroenteritis outbreaks in childcare centres, Victoria, Australia, 2012-2015. Epidemiol Infect. 2017;145(9):1933-41.

42. Rahman M, Rahman R, Nahar S, Hossain S, Ahmed S, Golam Faruque AS, et al. Norovirus diarrhea in Bangladesh, 2010-2014: prevalence, clinical features, and genotypes. J Med Virol. 2016;88(10):1742-50.

43. Mans J, Murray TY, Nadan S, Netshikweta R, Page NA, Taylor MB. Norovirus diversity in children with gastroenteritis in South Africa from 2009 to 2013 : Gll.4 variants and recombinant strains predominate. Epidemiol Infect. 2016; 144(5):907-16.

44. Makhaola K, Moyo S, Lechiile K, Goldfarb DM, Kebaabetswe LP. Genetic and epidemiological analysis of norovirus from children with gastroenteritis in Botswana, 2013-2015. BMC Infect Dis. 2018;18(1):246.

45. Wu X, Han J, Chen L, Xu D, Shen Y, Zha Y, et al. Prevalence and genetic diversity of noroviruses in adults with acute gastroenteritis in Huzhou, China, 2013-2014. Arch Virol. 2015;160(7):1705-13.

46. Chen C, Yan JB, Wang HL, Li P, Li KF, Wu B, et al. Molecular epidemiology and spatiotemporal dynamics of norovirus associated with sporadic acute gastroenteritis during 2013-2017, Zhoushan Islands, China. PLoS One. 2018; 13(7):e0200911.

47. Mahar JE, Kirkwood CD. Characterization of norovirus strains in Australian children from 2006 to 2008: prevalence of recombinant strains. J Med Virol. 2011;83(12):2213-9.

48. Phan TG, Kuroiwa T, Kaneshi K, Ueda Y, Nakaya S, Nishimura S, et al. Changing distribution of norovirus genotypes and genetic analysis of recombinant Gllb among infants and children with diarrhea in Japan. J Med Virol. 2006;78(7):971-8.

49. Bruggink LD, Moselen JM, Marshall JA. The comparative molecular epidemiology of GII.P7_Gll.6 and GII.P7_Gll.7 norovirus outbreaks in Victoria, Australia, 2012-2014. Intervirology. 2016;59(1):60-5.

50. Nagasawa K, Matsushima Y, Motoya T, Mizukoshi F, Ueki Y, Sakon N, et al. Phylogeny and immunoreactivity of norovirus GII.P16-GIl.2, Japan, winter 2016-17. Emerg Infect Dis. 2018;24(1):144-8.

51. Bidalot M, Théry L, Kaplon J, De Rougemont A, Ambert-Balay K. Emergence of new recombinant noroviruses GIl.p16-GIl.4 and Gll.p16-GIl.2, France, winter 2016 to 2017. Euro Surveill. 2017;22(15):30508.

52. Kwok K, Niendorf S, Lee N, Hung TN, Chan LY, Jacobsen S, et al. Increased Detection of Emergent Recombinant Norovirus GII.P16-GIl.2 Strains in Young Adults, Hong Kong, China, 2016-2017. Emerg Infect Dis. 2017;23(11):1852-5

53. Liu LT, Kuo TY, Wu CY, Liao WT, Hall AJ, Wu FT. Recombinant GII.P16-GII.2 norovirus, Taiwan, 2016. Emerg Infect Dis. 2017;23(7):1180-3.

54. Lu J, Fang L, Sun L, Zeng H, Li Y, Zheng H, et al. Association of GII.P16-GIl.2 recombinant norovirus strain with increased norovirus outbreaks, Guangdong, China, 2016. Emerg Infect Dis. 2017;23(7):1188-90.

\section{Publisher's Note}

Springer Nature remains neutral with regard to jurisdictional claims in published maps and institutional affiliations. 